\title{
Multi-View 3D Data Fusion for Visualization of the Inframammary Fold in Women with Ptotic Breasts
}

\author{
Lijuan ZHAO ${ }^{1}$, Gregory P. REECE ${ }^{2}$, Michelle C. FINGERET ${ }^{2,3}$, Fatima MERCHANTT ${ }^{* 1,4}$ \\ ${ }^{1}$ Department of Computer Science, University of Houston, Houston (TX), USA; \\ ${ }^{2}$ Department of Plastic Surgery, The University of Texas MD Anderson Cancer Center, Houston (TX), USA; \\ ${ }^{3}$ Dept. of Behavioral Science, The University of Texas MD Anderson Cancer Center, Houston (TX), USA; \\ ${ }^{4}$ Department of Engineering Technology, University of Houston, Houston (TX), USA
}

http://dx.doi.org/10.15221/14.029

\begin{abstract}
Three-dimensional (3D) imaging is finding increasing use in plastic surgery, both for breast reconstruction after oncologic surgery and for cosmetic augmentation/reduction procedures. The upright view image is conventionally used for surgical planning and outcome assessment. However, inframammary fold (IMF), a critical landmark for breast surgery and morphometry, is typically occluded from the upright view in women with ptotic breasts. In this study we used a custom designed tilt system (3dMD Inc., Atlanta, Georgia) that enables the capture of 3D images in both the upright and supine position. Our results demonstrate that for 3D images of ptotic breasts, wherein only the lowest contour of the breast touching the abdomen is visible, we can employ multi-view 3D image data fusion to superimpose the position of the anatomical IMF from the supine position onto the image in the position. This approach thus enables the physician to visualize the position of the IMF in the upright images of women with high degrees $(\geq 2)$ of breast ptosis.
\end{abstract}

Keywords: 3D image, upright and supine position, breast ptosis, inframammary fold, visualization

\section{Introduction}

The last decade has seen a steady increase in the use of 3D imaging for visualization and quantification of breast aesthetics in cosmetic and reconstructive breast surgery. 3D images of the female torso acquired in the upright position not only enable a $180^{\circ}$ panoramic visualization of the breasts, but also permit objective evaluation of breast aesthetics related to symmetry, projection, ptosis and volume. Objective measurements typically involve the use of anatomical landmarks (fiducial points) on the torso, such as the sternal notch, nipples, transition point and the IMF, and are performed on images acquired in the upright position.

An inherent limitation of photography is the inability to image areas that are occluded. Thus, when acquiring images of women with highly ptotic (sagging) breasts (ptosis grade $\geq 2$ ), the anatomical IMF is occluded and cannot be visualized in the upright position which is conventionally used for surgical planning and outcome assessment (see Fig .1). Consequently, it is impossible to delineate the anatomical IMF in the 3D images of women with ptotic breasts in an upright position, and we can only detect the lowest contour where the breast touches the abdomen (i.e. the contour of where the breast is touching the abdominal wall and is up under the breast in women with high degrees of ptosis but is visible if the image is rotated so that the viewer is looking from below at the breast).

The IMF is the actual fold under the breast that anatomically determines the lowest border of the base of the breast. In the upright position, only the medial and lateral parts are visible in women with high degrees of ptosis. However, delineation of the anatomical IMF on the upright view image is critical since the IMF is a defining element in the shape and structure of the female breast [1]. Evaluation of the IMF and its position is an important aesthetic consideration after breast reconstruction or augmentation [2]. The IMF is an important landmark that can be used to grade breast ptosis $[3,4]$ and facilitate volume calculation $[5,8,9]$. The distances from the sternal notch, nipple, and the lowest visible point of the breast, to the IMF are usually measured to evaluate breast morphology at a time point or to estimate changes in shape over time [4-12]. Typically, any changes in the IMF position are also quantified and compared at a time point or longitudinally along with analysis of breast and chest wall asymmetries $[6,10,11]$. Such estimates can be used during surgical planning to define and estimate breast morphology, and ultimately, impact patient satisfaction in cosmetic and reconstructive breast surgeries [7]. The IMF is not only an important anatomical landmark in breast reconstruction surgery $[1,2]$, but is also the preferred site for the access incision surgical approach in breast

*fmerchan@central.uh.edu; +1- 713- 743- 8292; www.tech.uh.edu/faculty/merchant/ 
augmentation [13]. It provides the best access with surgical control and direct visualization to the subglandular, subfascial, and subpectoral planes without violating the breast parenchyma during augmentation. However, in women with high degrees of ptosis $(\geq 2)$, the anatomical position of the IMF on the chest wall is occluded due to the sagging breast. In such cases, the anatomical IMF cannot be visualized in the upright position, and the physician has to manually lift the breast to localize the IMF. For computer based surgical planning, such manipulations are impossible in static images of the torso acquired in the upright position, and this limits the ability to perform a number of quantitative assessments involving the IMF on images of women with ptotic breasts [6, 12].

To overcome this problem, we propose a data fusion technique with 3D multi-view (upright and supine) images to visualize the anatomical IMF in an upright image.
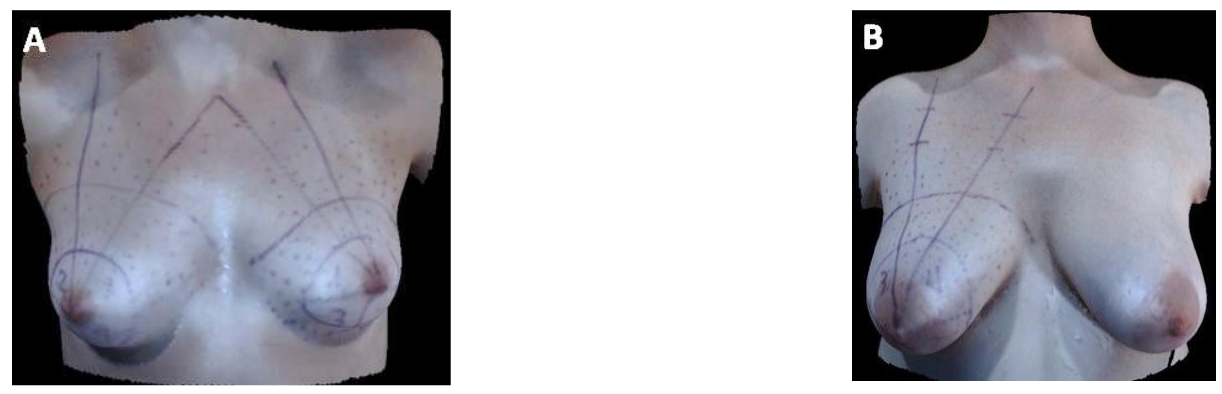

Fig. 1. (A) 3D image of breast with no ptosis, (B) 3D image of breast with degree 2 ptosis.

\section{Data acquisition}

Surface scans in the upright and supine positions were captured from commissioned female volunteers (IRB approved) using a custom designed tilt imaging system (3dMD Inc., Atlanta, Georgia). Surface images from five participants were used in this study. Age, race/ethnicity, body mass index (BMI), breast ptosis grade, and previous breast surgery information for these five participants are listed in Table 1.

Table 1. Demographics and characteristics of participants.

\begin{tabular}{|c|c|c|c|c|c|}
\hline Participants & Age & Race/Ethnicity & BMI & Ptosis grade & Previous breast surgery \\
\hline A & 21 & White/NH & 19 & 0 & None \\
\hline B & 53 & White/NH & 19.8 & 0 & Left breast TRAM flap \\
\hline C & 45 & White/NH & 27 & 3 & None \\
\hline D & 44 & White/NH & 27 & 2 & None \\
\hline E & 21 & White/H & 36 & 2 & None \\
\hline
\end{tabular}

Notes: NH: Non-Hispanic or Latino; H: Hispanic; TRAM, transverse rectus abdominis myocutaneous flap.

\section{Method}

The multi-view (upright and supine) images were processed as follows. First, we identified the points along the lowest contour of the breast touching the abdominal wall on the surface mesh in both the upright and supine images employing surface curvature analysis, using a modified version of the algorithm previously described in [14]. Cubic spline fitting of the identified points was then used to estimate the lowest breast contour. In this paper, we use the term "the lowest breast contour" to represent "the lowest contour where the breast touches the abdomen". Next we registered the upright and supine 3D images using a modified version of the algorithm previously described in [19], and employed the transformation parameters to superimpose the detected lowest breast contour from the supine image onto the upright image. The anatomical IMF is visible in the supine position for breasts, so the detected lowest breast contours in the supine images represent the anatomical IMF. Thus the fusion technique enables us to visualize the anatomical IMF position for ptotic breasts in the upright images.

\subsection{Detection of lowest breast contour}

We improved our previous contour detection algorithm, which employed Gaussian curvature analysis and random sample consensus (RANSAC) algorithm [14], to detect the lowest breast contours on 3D images [14]. The new method is described as follows. 


\subsubsection{Curvature analysis}

Curvature is defined as the amount that a surface deviates from being flat. At each point $p$ of a 3D surface one may find a normal plane, which contains the normal vector of the point $p$. The intersection of the normal plane and the 3D surface is a plane curve. The plane curves from different normal planes at point $p$ will generate different curvatures. The principal curvatures, $k_{\max }$ and $k_{\min }$, are the maximum and minimum values of the curvatures at $p$.

To calculate the principal curvatures on 3D surface mesh, we used a toolbox developed by Gabriel Peyre [15] based on the algorithms proposed by Cohen-Steiner et al. [16, 17]. The curvature tensor for each vertex was estimated using the following expression [17]:

$$
T(v)=\frac{1}{|B|} \sum_{\text {edges } e} \beta(e)|e \cap B| \bar{e} \bar{e}^{t}
$$

where $v$ is an arbitrary vertex on the 3D mesh, $|B|$ is the surface area around $v$ over which the curvature tensor is estimated, $\beta(e)$ is the signed angle between the normals to the two oriented triangles incident to edge $e$ (positive if convex, negative if concave), $|e \cap B|$ is the length of $e \cap B$ (always between 0 and $|e|$ ), and $\bar{e}$ is a unit vector in the same direction as $e$. The tensor is evaluated by approximating the neighborhood $B$ as the geodesic disk around this vertex. $D_{\max }$ and $D_{\min }$ are the two eigenvalues calculated from the tensor vector and provide estimates of principal curvatures at $v$. In this study, we employed a 10-ring neighborhood for $B$.

\subsubsection{Determination of reference points}

Before we detect the points along the lowest breast contour, we first located an initial estimate for the reference point $R P$ (see Fig. $2 \mathrm{C}$ ) of breast using shape index. $R P$ is estimated at the midpoint of the line joining the transition point and the nipple for each breast mound. It was used as a reference point to calculate angles of possible contour points in subsection 3.1.3. Shape index $S$ for each point on the surface mesh was given by equation 2 [18]. We employed a pseudo-color visualization method for viewing the shape index of the $3 \mathrm{D}$ mesh. Fig. $2 \mathrm{~A}$ presents a representative $3 \mathrm{D}$ upright image of the torso, and the color-mapped shape index for the torso is presented in Fig. 2B.

$$
S=\frac{2}{\pi} \tan ^{-1}\left(\frac{k_{\max }+k_{\min }}{k_{\max }-k_{\min }}\right)
$$

This algorithm is applicable to both breasts but for simplicity, is discussed here in terms of the right breast. The points on right half torso were divided into blocks (we used the block size as $5 \mathrm{~mm} X 5 \mathrm{~mm}$ by experience) based on their $x$ and $y$ coordinates ( $x, y$, and $z$ directions are displayed in Fig. 2A). For each block, the average of weighted shape index ave_S was computed, where the weights are $z$ coordinate of points in this block. We selected the block with the largest ave_S and the $x$ value of the center of this block is $x$ coordinate of $R P$. Then we searched a range (6X9 blocks) above this block and set the $y$ coordinate of $R P$ as $y$ value of center of the highest block with ave_S $>0$. The $6 X 9$ block region was empirically estimated to avoid $R P$ location lower than the lowest breast contour in images of ptotic breasts. The automatically estimated $R P$ locations for two breasts are showed in Fig. $2 \mathrm{C}$.
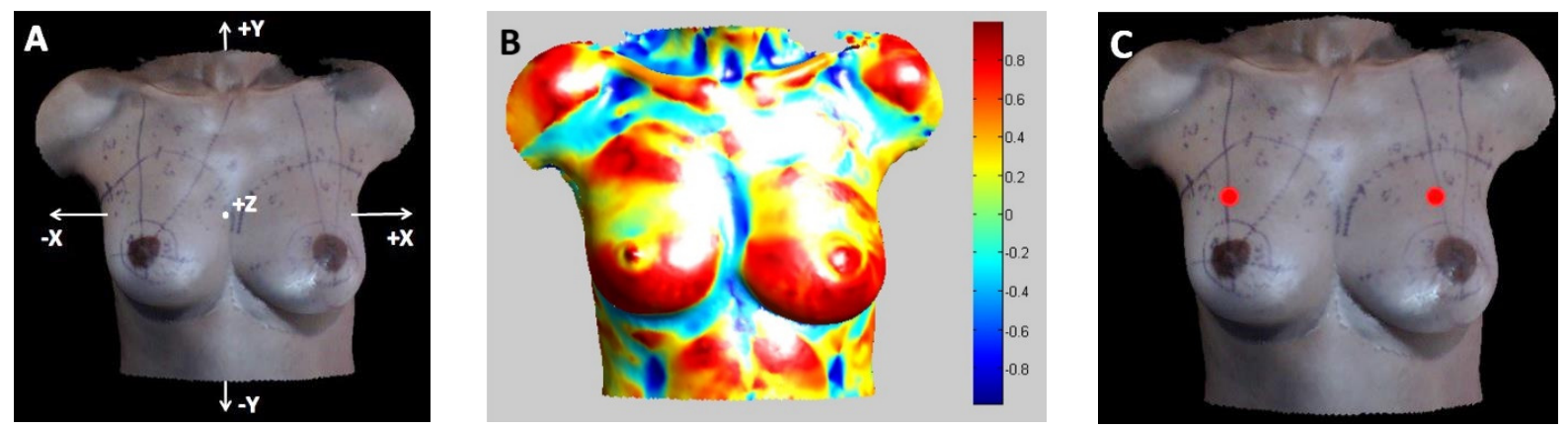

Fig. 2. (A) Representative 3D image of the upright female torso. The texture image is overlaid on the surface

(B) Color-mapped shape index of 3D surface scan, (C) Estimates of center points RPs (red) for the left and right breasts. 


\subsubsection{Lowest breast contour determination}

The points along the lowest breast contour were determined based on $R P$ location and a set of possible contour points $S P P$ (Fig. 3A). A possible contour point is a point which has negative shape index $S$ and $k_{\min }<$ mean of minimum principal curvatures for all points on the torso. The set of points in $S P P$ from the right half of the torso were sorted based on their angles relative to right $R P$ as defined by equation 3 :

$$
\theta=\operatorname{sign}\left(x_{P}-x_{R P}\right) \cos ^{-1}\left(\frac{\overrightarrow{v_{1}} \cdot \overrightarrow{v_{2}}}{\left|\overrightarrow{v_{1}}\right| \cdot\left|\overrightarrow{v_{2}}\right|}\right)
$$

Where $\overrightarrow{v_{1}}$ is a vector along $-y$ direction, $\overrightarrow{v_{2}}=\left(x_{P}-x_{R P}, y_{P}-y_{R P}\right)$ in which $x_{P}$ and $y_{P}$ are coordinates of a point $P$ in $S P P$ and $x_{R P}$ and $y_{R P}$ are coordinates of $R P$. First, we estimated an intermediate point of the contour which has minimum $k_{\min }$ value in $s P P$ points between angle interval $-2.5^{\circ} \sim 2.5^{\circ}$. Points in this interval are below $R P$ and have $x$ coordinates close to that of $R P$. Then we extended the contour points along two directions at angle interval $5^{\circ}$. An Interval of $5^{\circ}$ was empirically determined, to ensure that the detected contour points are not very sparse. In each interval we detected the contour point, such that it has a minimum $k_{\min }$ value, and the Euclidean distance to detected contour point in the previous interval $<2 L$, where $L$ is the arc length of current interval. If there is no $S P P$ point in an interval at distance $<2 L$, the detection was terminated in that direction. The detected lowest breast contour points are displayed in Fig. 3A. The resulting fitted cubic spline curve was identified as the lowest breast contour (Fig. 3B).
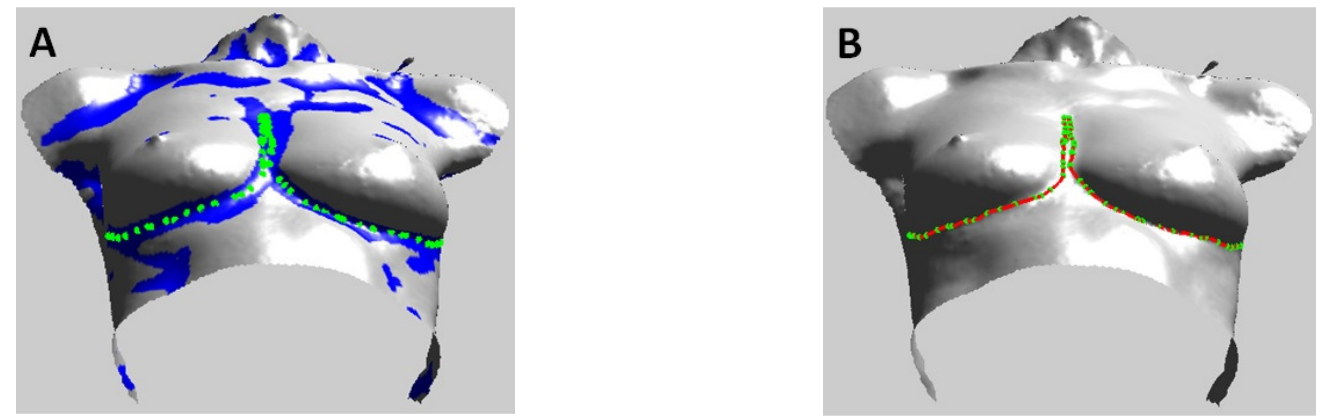

Fig. 3. (A) Set of possible contour points SPP (blue) and detected points along the lowest breast contours (green) displayed on surface, $(B)$ The detected lowest breast contours (red) exhibiting cubic spline curve fitting from the lowest breast contour points (green) displayed on surface.

\subsection{Registration of 3D torso images}

Our registration approach relies on the assumption that while the soft tissues of the patient's body may change over time, the skeleton is relatively stable. Thus the skeletal frame can be treated as being rigid, i.e. involving only translational and rotational transformations. Selecting points with reference to the skeletal frame and maximizing the correspondence between these points can then achieve 3D image registration.

\subsubsection{Control point selection}

We have previously reported a registration method for multiple-visit images during breast reconstruction [19], in which two fiducial points, the sternal notch (SN) and umbilicus (UM) were manually identified as two control points and other thirteen control points were automatically selected based on the location of SN and UM. In this study we adapted our previously developed registration algorithm for the transformation of 3D images taken in the supine position to an upright position, i.e. from $0^{\circ}$ to $90^{\circ}$. For supine to upright transformation of the female torso, the UM could not be used as a control point, since movement of the torso from the upright to the supine position is likely to result in the displacement of the soft tissues in the abdominal region which can result in some displacement of the UM position between the upright and supine position. Thus due to the non-rigidity of the UM position across the upright and supine position, in this study we used the midline point M positioned along the longitudinal (vertical) axis at the midpoint of the line joining the left and right nipples as the second control point (see Fig. 4). The algorithm is robust to discrepancies in the estimated point $M$ between different images, such as those caused by breast asymmetry in the same image, or breast 
shape changes in different images, due to the optimization process employed during the registration algorithm described in subsection 3.2.3. Let $d$ represent the straight line distance between SN and M. The other thirteen control points are equally spaced $(d / 6)$ along $x$ and $y$ directions. To improve precision, $z$ coordinates of the thirteen points were determined using linear interpolation in the surface mesh. Thus we obtained a total of fifteen control points.

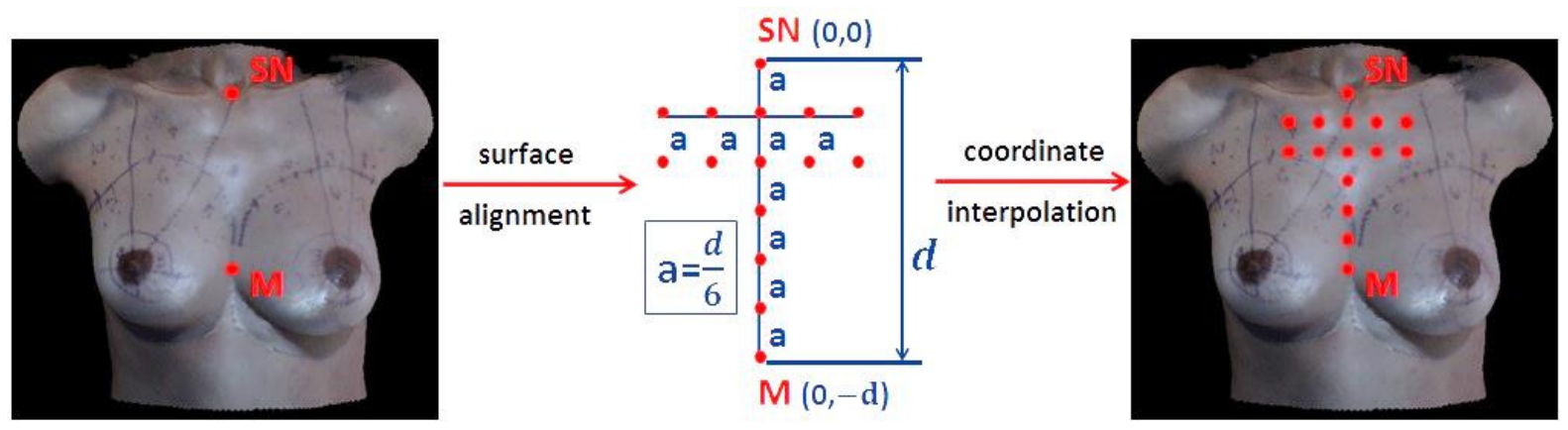

Fig. 4. Control points selected on the torso for $3 D$ correspondence

\subsubsection{Surface alignment}

Due to variations in participant pose and position during imaging, large discrepancies may tend to be introduced if we select the thirteen control points directly from the raw images acquired in the upright and supine position. To mitigate this effect, we initially aligned the longitudinal axis of the torso prior to the automated selection of the thirteen control points. Based on the coordinates of SN and M, we translated and rotated the torso image about the $z, x$, and $y$-axes such that $\mathrm{SN}$ is at the origin, the line joining SN-M is coincident to $y$-axis, and the surface is symmetric about $y z$ plane.

\subsubsection{Rigid registration}

For the fifteen points selected from each image, we created a complete graph in which each pair of points is connected by an edge. Images with upright and supine positions were then registered by determining the parameters for rigid transformation using the following cost function:

$$
f=\sum_{i=1}^{n} d_{i}^{2}+\frac{2}{(n-1)} \sum_{j=1}^{m}\left(\theta_{j} * l\right)^{2}
$$

Where $d_{i}$ is the Euclidean distance between the ith pair of control points in two images. $\theta_{j}$ is the angle between $j t h$ pair of edges in two complete graphs. $n=15 . m=C_{n}^{2}$, which is the number of edges in one complete graph, and $l$ is the average edge length for all edges in two complete graphs.

To avoid operator bias introduced by manual fiducial points identification and other discrepancies of identified $\mathrm{M}$ between the upright and supine images caused by breast shape changes, we optimized the registration of the $\mathrm{SN}$ and $\mathrm{M}$ by searching for the most optimal corresponding points within a pre-determined neighborhood of $50 \mathrm{~mm} X 50 \mathrm{~mm}$. The search region is $50 \mathrm{~mm}$ along $x$ and $y$ directions respectively. The control points in the first image (i.e. the upright image) were unchanged during optimization, whereas the control points in the second image (i.e. the supine image) were reselected based on the candidate locations of $S N$ and $M$ in each iteration. To obtain the best optimization, SN and M were optimized synchronously. For each iteration the 3D correspondence was determined by minimizing the cost function between control points from two images. The final $\mathrm{SN}$ and $\mathrm{M}$ locations and transformation parameters were obtained from the iteration with the minimum cost.

\subsection{Point fingerprints}

We evaluated our results by superimposing the detected lowest breast contours onto point fingerprints of the images. A point fingerprint is a set of $2 \mathrm{D}$ contours that are projections of geodesic circles onto a given tangent plane in local/global coordinate system [20]. In this study we used planes along the $z$-axis, which are parallel to the $x y$ plane to cut the surface to get a series of parallel circles. The point fingerprint contours were obtained by projecting the points of circles onto the $x y$ plane. 


\section{Results}

Results for five participants with varying degrees of ptosis are presented as follows, (i) No ptosis (Figs 5 \& 6), (ii) Ptosis grade of 2 (Figs 7 \& 8), and (iii) Ptosis grade of 3 (Fig 9). In Figs. 5-9, panels C and D show the detected lowest breast contours in the forward facing and backward facing views of the 3D upright images, respectively. Blue lines represent the lowest breast contours detected in the upright images. Red contours are the detected lowest breast contours transformed from the supine images and superimposed on the upright images. The anatomical IMF is visible in the images taken in the supine position for both non-ptotic and ptotic breasts. Thus the detected lowest breast contours in the supine images represent the anatomical IMF. Thus the red contours in Panels C-D in Fig. 5-9, represent the anatomical IMF from the supine images that is superimposed on the upright image to enable visualization of the anatomical IMF in the upright images. Fig. 5-9E show 2D projections of detected contours displayed on the fingerprint projection of the 3D upright images. Contours detected from the supine images are superimposed on the upright images. Fig. 5-9F show $2 \mathrm{D}$ fingerprint of $3 \mathrm{D}$ supine images with detected contours. Contours detected from the upright images are superimposed on the supine image.

As seen in Figs. 5-6, for non-ptotic breasts (ptosis degree $<1$ ), the anatomical IMF is visible in both the upright (Fig. 5-6A) and the supine (Fig. 5-6B) images, and coincides with the detected lowest breast contour. These data validate that the lowest breast contour detected in the supine image closely estimates the position of the anatomical IMF.

For a ptosis grade of 2, the anatomical IMF is occluded in the upright images (Fig. 7-8A), but can be visualized in the supine images (Fig. 7-8B). As seen in Fig. 7-8, C-F, the lowest breast contours, i.e. anatomical IMF (red), detected from the supine image is higher than the lowest breast contour (blue) that is detected from the upright image. These results demonstrate that $3 \mathrm{D}$ data fusion of information from the supine images can be used to visualize structures that are occluded from the upright images.

For breasts of ptosis grade 3 in Fig. 9, the anatomical IMF is also occluded in the upright image (Fig. 9A), but can be visualized in the supine image (Fig. 9B). Due to data missing during image acquisition along the lowest contour area of left breast for the upright image (Fig. 9A), we were unable to run the proposed algorithm to estimate the lowest breast contour of the left breast. But the detected lowest contours of right breasts for both the upright and supine images are presented in Fig. 9C-F, and demonstrate that the 3D data fusion technique can be used to visualize the occluded IMF in the upright images of women with high ptosis.

\section{Conclusions}

We have designed a data fusion technique with 3D multi-view (upright and supine) images to visualize the IMF which is typically occluded from the upright view for women with ptotic breasts. The detected lowest contour of the breast touching the abdomen in the supine image (that represents the anatomical IMF) was transformed and superimposed onto the upright image. Our experimental results on non-ptotic breasts demonstrated that the lowest breast contour detected in the supine image closley estimates the position of the anatomical IMF in the upright image. And the results on breasts of ptosis grades 2 and 3 validated that the lowest breast contour detected in the supine image (the anatomical IMF) could be used to visualize the anatomical IMF position for ptotic breasts in the upright image. The upright view image is conventionally used for surgical planning and outcome assessment in plastic surgery, both for breast reconstruction after oncologic surgery and for cosmetic procedures such as breast augmentation or reduction. An inherent limitation of the 3D upright view image is the inability to image areas that are occluded. Thus, some important landmarks and structures (e.g. the anatomical IMF) are occluded and cannot be visualized in the upright position. However, delineation of the anatomical IMF is critical since its position is an important consideration for both aesthetic outcome evaluation and surgical planning in plastic surgery, and will ultimately serve to optimize patient satisfaction in cosmetic and reconstructive breast surgeries. The proposed data fusion technique with 3D multi-view (upright and supine) images to visualize the IMF in the upright image is a critical landmark for computational screening and planning in breast surgery, that has potential for clinical realization in quantitative breast morphometry. 


\section{Acknowledgements}

This work was supported by a NIH grant 1R01CA143190-01A1 (PI: Mia Markey). The data used in this study were generously provided by the Department of Plastic Surgery at The University of Texas MD Anderson Cancer Center.
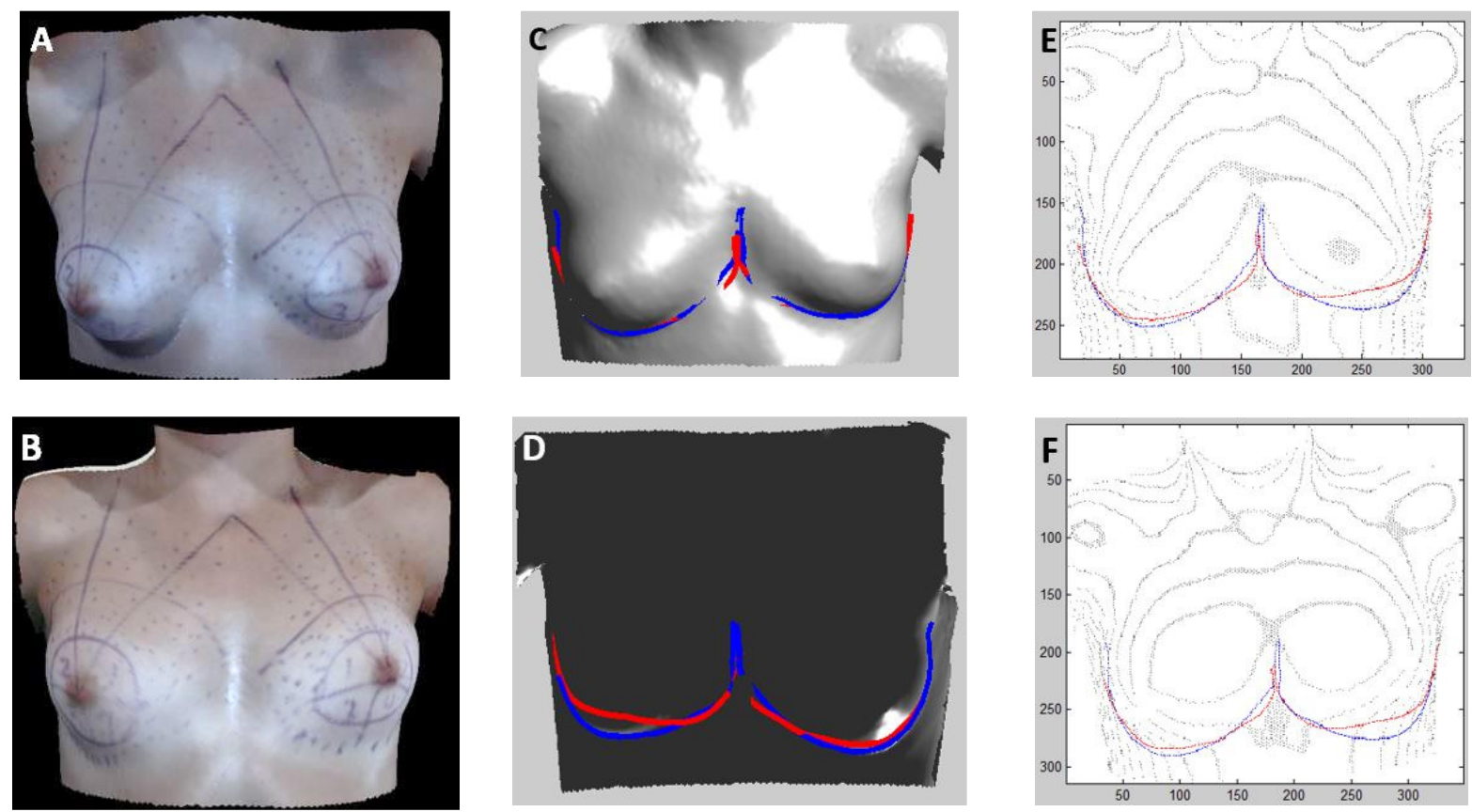

Fig. 5. Subject with no ptosis. (A) 3D image in the upright position, (B) 3D image in the supine position, (C) The detected lowest breast contours displayed in the upright image (front), (D) The detected lowest breast contours displayed in the upright image (back), (E) 2D projection of detected contours displayed on fingerprint projection of the upright image, $(F)$ 2D projection of detected contours displayed on fingerprint projection of the supine image. Blue lines represent the lowest breast contours detected in the upright images, whereas red lines are the lowest breast contours detected from the supine image (i.e. the anatomical IMF).
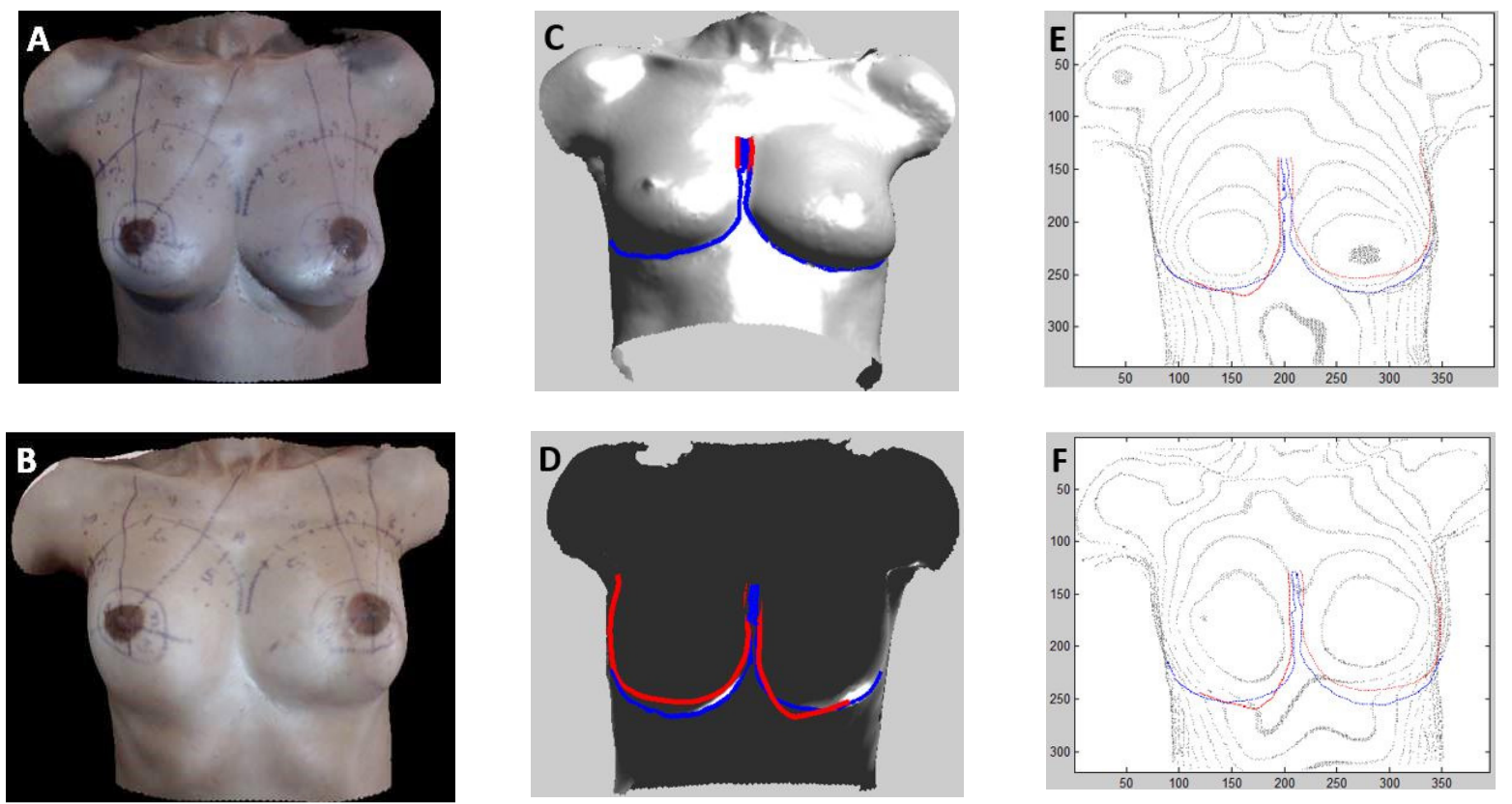

Fig. 6. Another subject with no ptosis. (A) 3D image in the upright position, (B) 3D image in the supine position, (C) The detected lowest breast contours displayed in the upright image (front), (D) The detected lowest breast contours displayed in the upright image (back), (E) 2D projection of detected contours displayed on fingerprint projection of the upright image, $(F)$ 2D projection of detected contours displayed on fingerprint projection of the supine image. Blue lines represent the lowest breast contours detected in the upright images, whereas red lines are the lowest breast contours detected from the supine image (i.e. the anatomical IMF). 

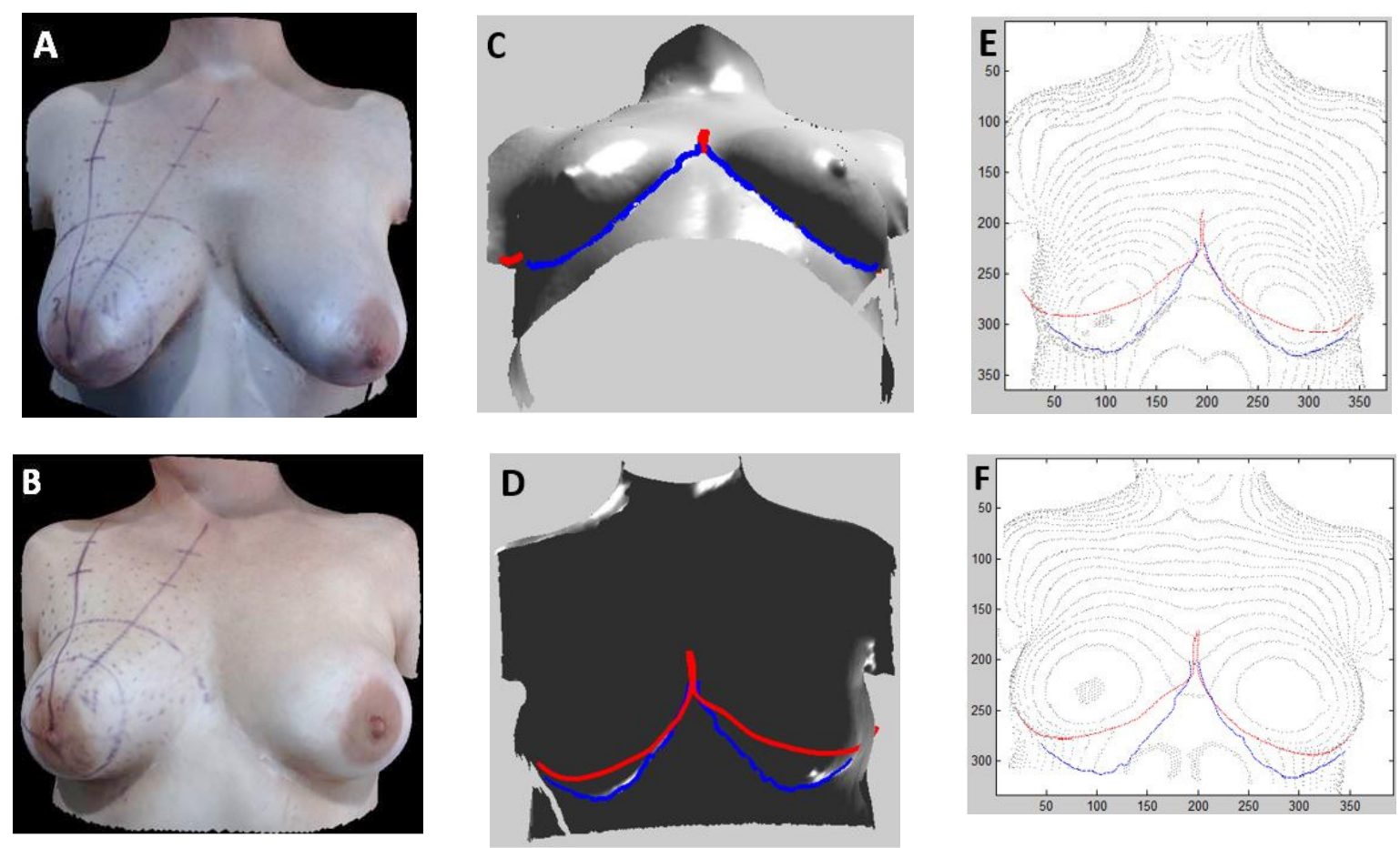

Fig. 7. Subject with moderate ptosis (Grade 2). (A) 3D image in the upright position, (B) 3D image in the supine position, (C) The detected lowest breast contours displayed in the upright image (front), (D) The detected lowest breast contours displayed in the upright image (back), (E) 2D projection of detected contours displayed on

fingerprint projection of the upright image, $(F)$ 2D projection of detected contours displayed on fingerprint projection of the supine image. Blue lines represent the lowest breast contours detected in the upright images, whereas red lines are the lowest breast contours detected from the supine image (i.e. the anatomical IMF).
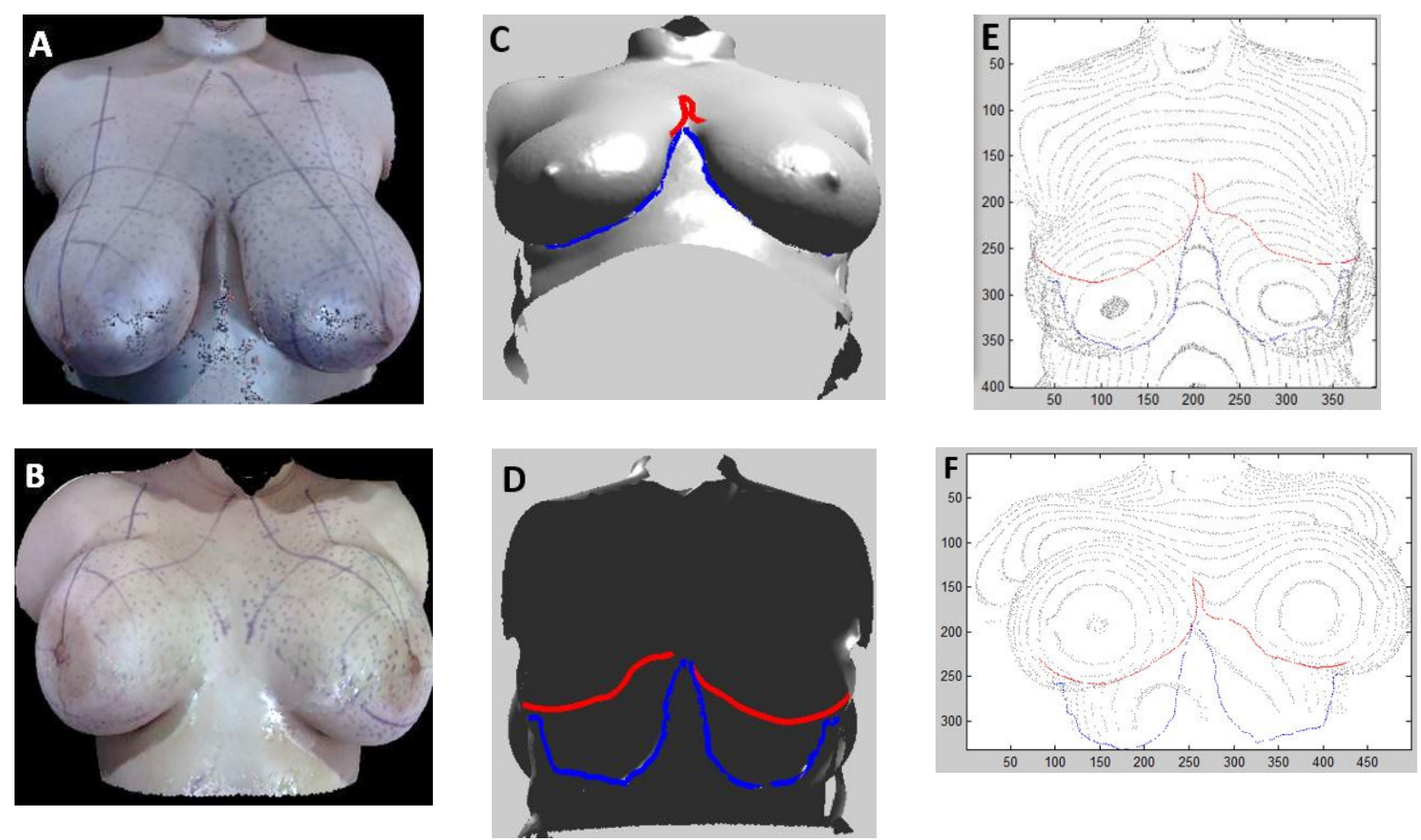

Fig. 8. Another subject with moderate ptosis (Grade 2). (A) 3D image in the upright position, (B) $3 D$ image in the supine position, (C) The detected lowest breast contours displayed in the upright image (front), (D) The detected lowest breast contours displayed in the upright image (back), $(E)$ 2D projection of detected contours displayed on fingerprint projection of the upright image, $(F) 2 D$ projection of detected contours displayed on fingerprint projection of the supine image. Blue lines represent the lowest breast contours detected in the upright images, whereas red lines are the lowest breast contours detected from the supine image (i.e. the anatomical IMF). 

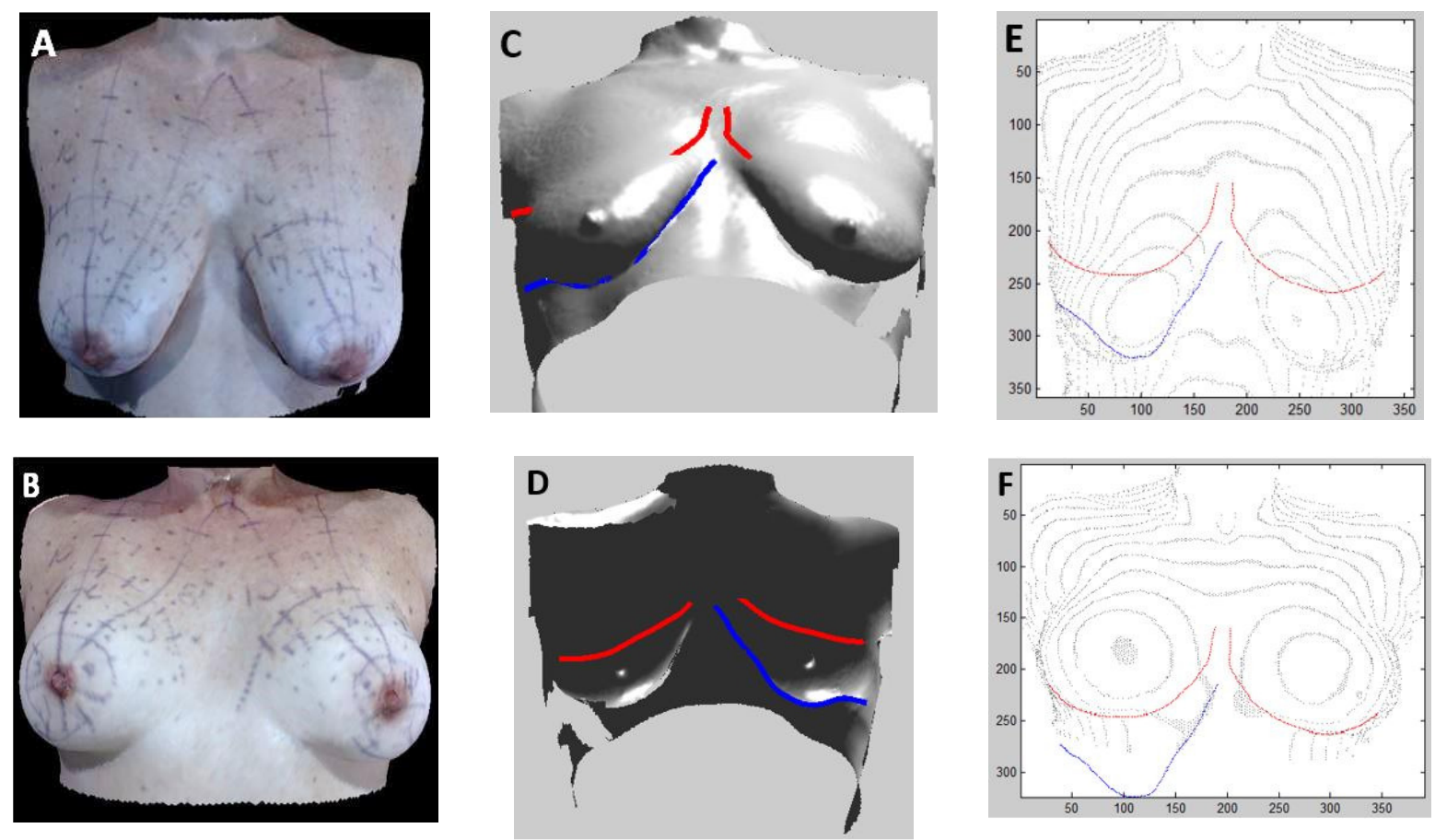

Fig. 9. Subject with high ptosis (Grade 3). (A) 3D image in the upright position, (B) 3D image in the supine position, (C) The detected lowest breast contours displayed in the upright image (front), (D) The detected lowest breast contours displayed in the upright image (back), (E) 2D projection of detected contours displayed on

fingerprint projection of the upright image, $(F) 2 D$ projection of detected contours displayed on fingerprint projection of the supine image. Blue lines represent the lowest breast contours detected in the upright images, whereas red lines are the lowest breast contours detected from the supine image (i.e. the anatomical IMF). The lowest contour of left breast for upright image is missed due to data missing on this area.

\section{References}

[1] C. D. Muntan, M. J. Sundine, R. D. Rink, and R. D. Acland, "Inframammary Fold: A Histologic Reappraisal", in Plastic and Reconstructive Surgery, Vol. 105, No. 2, 2000, pp. 549-556, http://dx.doi.org/10.1097/00006534-200002000-00011

[2] G. P. Gui, K. A. Behranwala, N. Abdullah, J. Seet, P. Osin, A. Nerurkar, and S. R. Lakhani, "The Inframammary Fold: Contents, Clinical Significance and Implications for Immediate Breast Reconstruction", in British Journal of Plastic Surgery, Vol. 57, No. 2, 2004, pp. 146-149, http://dx.doi.org/10.1016/j.bjps.2003.11.030

[3] M. S. Kim, G. P. Reece, E. K. Beahm, M. J. Miller, E. N. Atkinson, and M. K. Markeyemail, "Objective Assessment of Aesthetic Outcomes of Breast Cancer Treatment: Measuring Ptosis from Clinical Photographs", in Computers in Biology and Medicine, Vol. 37, No. 1, 2007, pp. 49-59, http://dx.doi.org/10.1016/j.compbiomed.2005.10.007

[4] Y. J. Liu, "Aesthetics of the Female Breast: Correlation of Pluralistic Evaluations with Volume and Surface Area", in Yale Journal of Biology and Medicine, Vol. 82, No. 4, 2009, pp. 181-229

[5] C. N. Creasman, D. Mordaunt, T. Liolios, C. Chiu, A. Gabriel, and G. P. Maxwell, "Four-Dimensional Breast Imaging, Part I: Introduction of a Technology-Driven, Evidence-Based Approach to Breast Augmentation Planning", in Aesthetic Surgery Journal, Vol. 31, No. 8, 2011, pp. 914-924, http://dx.doi.org/10.1177/1090820X11423916

[6] C. N. Creasman, D. Mordaunt, T. Liolios, C. Chiu, A. Gabriel, and G. P. Maxwell, "Four-Dimensional Breast Imaging, Part II : Clinical Implementation and Validation of a Computer Imaging System for Breast Augmentation Planning", in Aesthetic Surgery Journal, Vol. 31, No. 8, 2011, pp. 925-938, http://dx.doi.org/10.1177/1090820X11424147

[7] Y. J. Liu and J. G. Thomson, "Ideal Anthropomorphic Values of the Female Breast Correlation of Pluralistic Aesthetic Evaluations With Objective Measurements", in Annals of plastic surgery, Vol. 67, No. 1, 2011, pp. 7-11, http://dx.doi.org/10.1097/SAP.0b013e3181f77ab5 
[8] M. Eder, M. Klöppel, D. Müller, N. A. Papadopulos, H. G. Machens, and L. Kovacs, "3-D Analysis of Breast Morphology Changes After Inverted T-Scar and Vertical-Scar Reduction Mammaplasty Over 12 Months", in Journal of Plastic, Reconstructive \& Aesthetic Surgery, Vol. 66, No. 6, 2013, pp. 776-786, http://dx.doi.org/10.1016/j.bjps.2013.02.008

[9] L. Kovacs, M. Eder, R. Hollweck, A. Zimmermann, M. Settles, A. Schneider, M. Endlich, A. Mueller, K. Schwenzer-Zimmerer, N. A. Papadopulos, and E. Biemer, "Comparison Between Breast Volume Measurement Using 3D Surface Imaging and Classical Techniques", in Breast, Vol. 16, No. 2, 2007, pp. 137-145, http://dx.doi.org/10.1016/i.breast.2006.08.001

[10]L. Kovacs, M. Eder, A. Zimmermann, D. Müller, T. Schuster, N. A. Papadopulos, E. Biemer, M. Klöppel, and H. G. Machens, "Three-Dimensional Evaluation of Breast Augmentation and the Influence of Anatomic and Round Implants on Operative Breast Shape Changes", in Aesthetic Plastic Surgery, Vol. 36, No. 4, 2012, pp.879-887, http://dx.doi.org/10.1007/s00266-012-9892-3

[11]M. Eder, F. V. Waldenfels, M. Sichtermann, T. Schuster, N. A. Papadopulos, H. G. Machens, E. Biemer, and L. Kovacs, "Three-Dimensional Evaluation of Breast Contour and Volume Changes Following Subpectoral Augmentation Mammaplasty Over 6 Months", in Journal of Plastic, Reconstructive \& Aesthetic Surgery, Vol. 64, No. 9, 2011, pp. 1152-1160, http://dx.doi.org/10.1016/j.bjps.2011.03.037

[12] J. Lee, M. Kawale, F. A. Merchant, J. Weston, M. C. Fingeret, D. Ladewig, G. P Reece, M. A. Crosby, E. K. Beahm, and M. K. Markey, "Validation of Stereophotogrammetry of the Human Torso", in Breast cancer: basic and clinical research, Vol. 5, 2011, pp. 15-25, http://dx.doi.org/10.4137/BCBCR.S6352

[13] B. S. Atiyeh, S. A. Dibo, M. Nader, and N. J. Papazian, "Preoperative Assessment Tool for the Planning of Inframammary Incision and Implant Profile in Breast Augmentation", in Aesthetic Plastic Surgery, July 25, 2014, pp. 1-9, http://dx.doi.org/10.1007/s00266-014-0381-8

[14]L. Zhao, S. K. Shah, G. P. Reece, M. A. Crosby, M. C. Fingeret, and F. A. Merchant, "Automated Detection of Breast Contour in 3D Images of the Female Torso", in Proc. of 4th International Conf. on 3D Body Scanning Technologies, Long Beach CA, USA, 2013, pp. 273-278, http://dx.doi.org/10.15221/13.273

[15] G. Peyre, Toolbox Graph, http://www.mathworks.com/matlabcentral/fileexchange/5355-toolbox-graph, accessed 2013

[16] D. Cohen-Steiner and M. Jean-Marie, "Restricted Delaunay Triangulations and Normal Cycle", in Proc. of 19th Conf. on Computational Geometry (SCG-03), New York, USA, 2003, pp. 237-246, http://dx.doi.org/10.1145/777792.777839

[17]P. Alliez, D. Cohen-Steiner, O. Devillers, B. Lévy, and M. Desbrun, "Anisotropic Polygonal Remeshing", in ACM Transactions on Graphics, Vol. 22, No. 3, 2003, pp. 485-493, http://dx.doi.org/10.1145/882262.882296

[18] H. Cantzler and R. B. Fisher, "Comparison of HK and SC Curvature Description Methods", in Proc. of $3^{\text {rd }}$ International Conf. on 3-D Digital Imaging and Modelling, Quebec City, Canada, 2001, pp. 285-291, http://dx.doi.org/10.1109/IM.2001.924458

[19] L. Zhao, S. K. Shah, G. P. Reece, M. A. Crosby, E. K. Beahm, M. C. Fingeret, M. K. Markey, and F. A. Merchant, "Semi-Automated Registration of 3D Torso Images from Breast Reconstruction Surgery", in Proc. of $3^{\text {rd }}$ International Conf. on 3D Body Scanning Technologies, Lugano, Switzerland, 2012, pp. 179-188, http://dx.doi.org/10.15221/12.179

[20]Y. Sun, J. Paik, A. Koschan, D. L. Page, and M. A. Abidi, "Point Fingerprint: A New 3-D Object Representation Scheme", in IEEE Transactions on Systems, Man, and Cybernetics, Part B: Cybernetics, Vol. 33, No. 4, 2003, pp. 712-717, http://dx.doi.org/10.1109/TSMCB.2003.814295 